\title{
Sistemas de Soporte de Decisión Espacial para Evaluaciones Ecológicas de Paisaje en el Estudio de Viabilidad del Suroeste de la Florida1
}

\author{
Leonard Pearlstine, Donald L. DeAngelis, Frank Mazzotti, Tomma Barnes, Michael Duever, Janet \\ Starnes y Marianna D. Conill ${ }^{2}$
}

\section{Introducción}

El objetivo del Estudio de Viabilidad del Suroeste de la Florida (SWFFS, por sus siglas en inglés) es desarrollar un plan regional de sondeo comprensivo y ecológico para los recursos acuáticos en la Florida del suroeste. Los límites del estudio se muestran en la figura 1. El área de estudio del SWFFS cubre alrededor de 4,300 millas cuadradas, y se ubica al oeste de los Everglades y de todas las actividades del Plan Comprensivo de la Restauración de los Everglades (CERP, por sus siglas en inglés). El estudio fue recomendado por CERP y el Estudio de Revisión Comprensiva para ser incluido en el Proyecto de la Florida Central y del Sur (C\&SF, por sus siglas en inglés) por: (1) asuntos ecológicos y de suministro de agua asociados con el suministro de aguas del Lake Okeechobee hacia el Caloosahatchee River, y porque (2) las alteraciones hidrológicas en tierra firme tienen efectos substanciales tanto existentes como potenciales sobre los recursos naturales y la biodiversidad presentes dentro del área de estudio. Este estudio tratará la salud de los ecosistemas terrestres y acuáticos y considerará una variedad de parámetros que incluyen flujo de agua, calidad de agua, suministro de agua, mantenimiento de la protección del flujo existente, fauna silvestre, diversidad biológica y hábitat natural. El SWFFS es conducido por el U.S. Army Corps of Engineers y el South Florida Water Management District. El USGS, en cooperación con University of Florida, provee asistencia técnica para la evaluación de los impactos hidrológicos sobre sistemas naturales.

La toma de decisiones a nivel de paisaje es un proceso que envuelve múltiples objetivos, grandes series de datos y muchas dudas e incertidumbres. Para respaldar este proceso, se necesitan herramientas de evaluación para hacer decisiones informadas y de largo plazo acerca de los recursos regionales y para

1. Este documento es Cir 1479S, perteneciente a la serie del Departamento de Ecología y Conservación de Fauna Silvestre, University of Florida, Cooperative Extension Service, Institute of Food and Agricultural Sciences. Fecha de publicación: Enero, 2006. Visite la página web http://edis.ifas.ufl.edu. Imprímase a color para mejor comprensión de gráficos y figuras.

2. Leonard Pearlstine, Frank J. Mazzotti y Marianna Domínguez Conill, University of Florida, Ft. Lauderdale Research and Education Center, 3205 College Ave, Ft. Lauderdale, Florida, 33314, e-mails: pearlstn@ufl.edu, fjma@ufl.edu, merrydm@ @otmail.com. Donald L. DeAngelis, U.S. Geological Survey, Florida Integrated Science Center, Water and Restoration Studies, University of Miami, P.O. Box 249118, Coral Gables, FL 33124, e-mail: ddeangelis@umiami.ir.miami.edu. Tomma Barnes, Michael Duever y Janet Starnes, South Florida Water Management District, Lower West Coast Regional Service Center, 2301 McGregor Boulevard, Ft Myers, FL 33901, e-mails: tbarnes@sfwmd.gov, mduever@sfwmd.gov, jstarne@sfwmd.gov. 


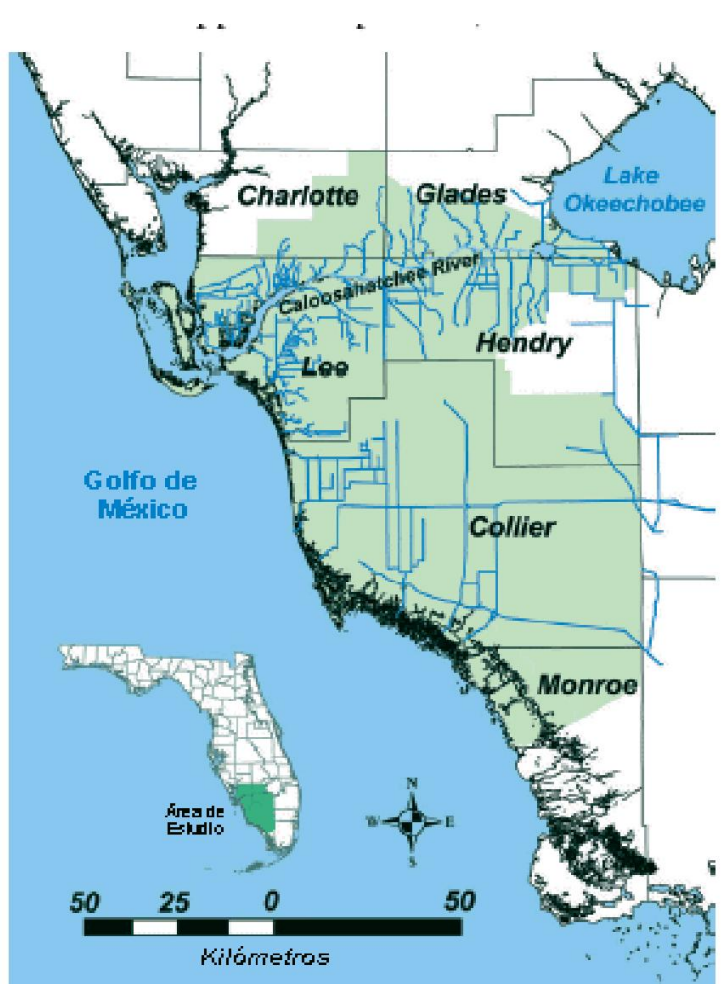

Figura 1. Área del Estudio de Viabilidad del Suroeste de la Florida (mostrada en verde) mostrando los canales principales.

reconocer las necesidades de investigación. Estas herramientas pueden ayudar a las autoridades involucradas en la restauración ecológica identificando las variables de decisión, desarrollando heurísticas de resolución de problemas, y evaluando las consecuencias de acciones de políticas alternas. El sistema de soporte de decisión espacial (SDSS, por sus siglas en inglés) para el manejo de recursos naturales es una herramienta computarizada que integra estrechamente modelos de teoría de decisión con modelos ecológicos y con análisis y mapeo de Sistemas de Información Geográfica (GIS, por sus siglas en inglés). La información proveída por SDSS ofrece a los encargados de la toma de decisiones una mayor habilidad para seguir los resultados de variables que interactúan, mejora la reproducibilidad de decisiones, y documenta las razones por las cuales (con alternativas conflictivas) fue tomada una decisión en particular (Rauscher 1999).

El sistema para el soporte de decisiones de evaluaciones de hábitat a nivel de paisaje presenta cuatro elementos (Fig. 2). Los primeros dos elementos, principalmente dirigidos hacia el científico, asisten en la toma de decisiones acerca de cuál criterio define mejor las medidas de rendimiento para la evaluación ecológica y cuáles valores tendrán esos criterios en un panorama en particular. El primer elemento es un análisis de los panoramas propuestos y de sus respectivos efectos en el ambiente físico. Estos análisis son apoyados por los datos de entrada provenientes de modelos que simulan cada panorama tales como modelos hidrológicos, modelos de crecimiento urbano, y modelos de calidad de agua. Las herramientas proveídas en el segundo elemento evalúan los efectos sobre el hábitat de fauna silvestre y sobre las comunidades ecológicas ocasionados por cambios en el ambiente físico. Estas herramientas evalúan la idoneidad de hábitat y el riesgo de las evaluaciones basadas en los datos de entrada de los modelos de hábitat.

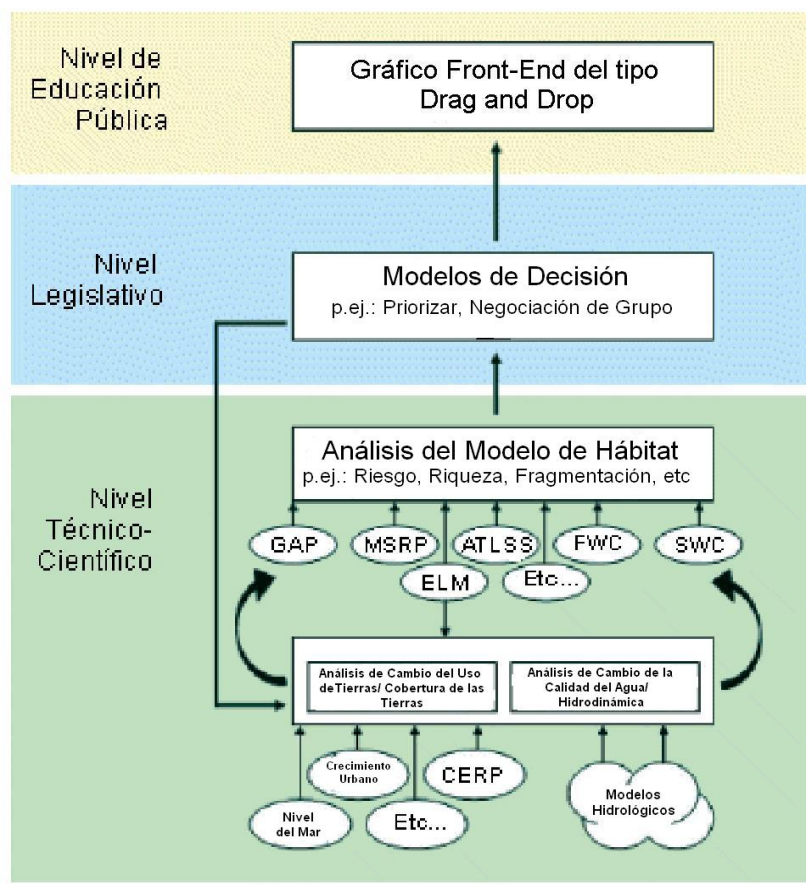

Figura 2. Un planteamiento jerárquico y modular para el desarrollo de un Sistema de Soporte de Decisión Espacial. Las abreviaciones representan modelos de hábitat actuales tanto para el sur como para el suroeste de la Florida.

Los elementos tercero y cuarto tienen la intención de ser utilizados por los legisladores y por el público, respectivamente. Los modelos de decisión (el tercer elemento) clasifican y asisten las decisiones entre los criterios y los panoramas alternativos. Las evaluaciones de las alternativas a 
este nivel pueden conllevar a panoramas nuevos o modificados, que luego serían entregados nuevamente a los científicos para ser evaluados utilizando las herramientas de soporte en los primeros dos componentes. Una versión educativa gráfica y pública (el último elemento) puede aumentar el entendimiento público acerca de las acciones de conservación, crear un sentido de envolvimiento y de "pertenencia" en las decisiones, y alertar a los legisladores y a los gerentes de recursos acerca del juicio social respecto a los planes alternativos. Una interfase diseñada para navegadores de la red de Internet es una opción para la participación del público y de los legisladores. El Internet tiene las ventajas de la remoción de restricciones geográficas para la participación, el fácil acceso a materiales de discusión, la introducción de datos anónimamente y la oportunidad de obtener reacciones sin confrontaciones (Kingston y otros, 2000).

\section{Asuntos Ecológicos en la Florida del Suroeste}

Las cuencas de Big Cypress y Caloosahatchee que conforman el SWFFS incluyen estuarios costeros dominados por manglar- y fanerógamas marinas. La productividad primaria, basada en el bentos, de estos protegidos criaderos mantienen comunidades de peces y de microinvertebrados que, a su vez, sirven de alimento para muchas especies marinas de uso comercial y recreativo. Junto a estas comunidades costeras, los pantanos de agua dulce y de cortos hidroperíodos en tierra firme, las sabanas húmedas y los bosques de humedales conforman el hábitat para 20 especies listadas Federalmente y 43 especies de aves no migratorias que no son de cacería y que son de especial preocupación por su manejo (U.S. Department of the Interior, 2004). La Florida del suroeste también representa una de las regiones más importantes para el soporte de especies que se movilizan en grandes áreas tales como la pantera de Florida, el oso negro de Florida y la cigüeña (Cox y otros 1994). Como el suroeste de la Florida es una de las áreas de más rápido desarrollo en la Nación, ha surgido la preocupación entre el público y los encargados de hacer decisiones acerca del crecimiento urbano y agrícola en las proximidades de extensas tenencias de tierras públicas y áreas naturales privadas con los atributos ecológicos antes descritos.

\section{Evaluaciones Ecológicas en el Suroeste de la Florida}

La siguiente discusión provee ejemplos de los tipos de herramientas y procedimientos que están siendo desarrollados para las evaluaciones en el suroeste de la Florida. El SWFFS es un proyecto progresivo, y las herramientas pueden cambiar substancialmente en el transcurso del estudio.

Las evaluaciones ecológicas examinan los efectos del cambio hidrológico en dos regiones geográficas. En zonas costeras, los cambios de flujo en el horario de suministro de agua a lo largo del Caloosahatchee River son evaluados por su efecto en el estuario del Caloosahatchee River. En zonas interiores, los cambios en las características hidrológicas terrestres son evaluados por su efecto en humedales boscosos y emergentes.

Se están desarrollando criterios para evaluar los efectos ecológicos sobre la Florida del suroeste a tres escalas principales. Los criterios tratan cambios en: (1) distribución y calidad potencial de hábitat para especies individuales, (2) composición de la comunidad y características hidrológicas, y (3) conectividad del paisaje.

\section{Evaluaciones de Especies}

Las herramientas de evaluación de especies costeras incluyen modelos de idoneidad de hábitat para la trucha marina, la ostra Americana, el cangrejo azul, dos especies de fanerógamas marinas (Halodule y Thalassia), y una gramínea sumergida de agua dulce (Vallisneria). Modelos de evaluación tierrestre están siendo creados o adaptados a partir de modelos existentes para indicadores de la salud de humedales internos, incluyendo:

- Vadeadores (Ibis Blanco y Cigüeñas)

- Anfibios (cambios en la composición de las comunidades de ranas)

- Fauna acuática (cambios en la composici[on de las comunidades de cangrejos de río y peces de forrajeo) 
- Medidas de la fragmentación de hábitats regionales

- Medidas de la conectividad de hábitats regionales para animales que se desplazan por grandes áreas

- Medidas de gradientes hidrológicos

La figura 3 muestra los resultados de un modelo de hábitat de especies para la trucha pinta en San Carlo Bay y Pine Island Sound en la boca del Caloosahatchee River. Se muestran los mapas de hábitat potencial para la trucha pinta para dos horarios de liberación de agua alternativos a lo largo del Caloosahatchee River. La interfase espacialmente-explícita ayuda al personal y a los encargados de la toma de decisiones a entender cómo se derivan los puntajes de los hábitas y a entender la distribución de los hábitats preferidos a lo largo del área de estudio. Además de mapear los puntajes de los hábitats, los puntajes de las componentes individuales, tales como salinidad y flujo, son mapeadas para que los usuarios puedan determinar rápidamente su contribución al puntaje total. En el cuadro superior se muestra la visualización mensual. Cada panorama se muestra como un grupo de pantallas espaciales que mapean la distribución de valores del Índice de Idoneidad de Paisaje (HSI, por sus siglas en inglés) para esa especie, durante ese mes, y la distribución de valores para cada componente que conllevan a los puntajes de HSI. Los cuadros de diálogo dentro de cada pantalla muestran valores en localidades seleccionadas por el usuario haciendo clic en el mapa. La pantalla de abajo representa la misma interfase cuando se muestran los totales anuales. En este caso, cuando los usuarios seleccionan una localidad haciendo clic sobre la misma, el valor HSI y todos los valores de las componentes que conforman ese HSI en esa localidad son graficados para cada mes de ese año.

\section{Evaluaciones de Comunidad}

Las evaluaciones de comunidad involucran el uso de modelos hidrológicos para evaluar las características hidrológicas dentro de las regiones indicadoras en el área de estudio. Las regiones indicadoras son áreas representativas de una única comunidad tales como los montes húmedos de pinos

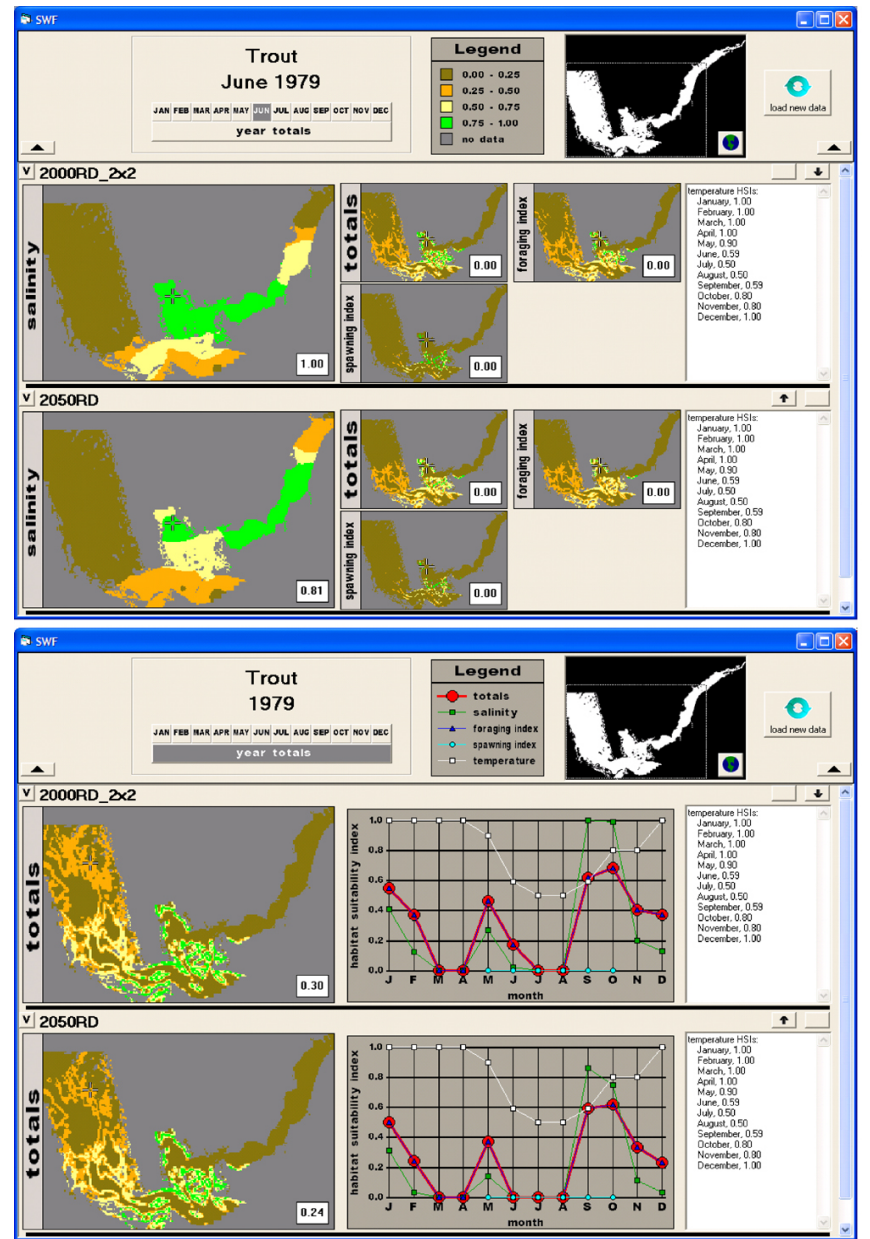

Figura 3. La interfase del Estudio de Viabilidad del Suroeste de la Florida mostrando la idoneidad de hábitat para la trucha pinta.

o las praderas de ciprés. Las características hidrológicas pre-desarrollo modeladas en una región indicadora son comparadas con las mismas características en panoramas hidrológicos alternos utilizando una medida de similitud. Las alternativas que mejor preserven la hidrología pre-desarrollo de una comunidad son categorizadas como las mejores para el mantenimiento de las condiciones naturales en ese sitio en particular.

\section{Evaluaciones de Paisaje}

La conectividad de paisaje es una medida de cómo los elementos del paisaje, tales como parches de hábitat, límites y contiguiidad, afectan los movimientos de los organismos y el uso de los recursos del paisaje (Tischendorf y Fahrig, 2000). El Oso Negro de la Florida está siendo utilizado como una especie paraguas, ya que sus requerimientos de conectividad a lo largo del paisaje protegen los 
requerimientos de hábitat para otras especies que también necesitan grandes áreas para su desplazamiento (p. ej. Pantera de la Florida, Lince Rojo). El mantenimiento de la conectividad requiere grandes áreas contiguas del hábitat preferido por el animal que estén conectadas por coberturas de tierra atravesables. Las grandes áreas centrales de hábitat preferido deben estar dentro de la distancia de dispersión del Oso Negro.

Otras medidas del paisaje incluyendo los tamaños de los parches de hábitat, densidad de carreteras, cantidad de borde del hábitat, la distribución de humedales de corto a largo hidroperíodo, y la distribución de las distancias de humedales a altiplanicies, contribuyen a la caracterización de la calidad regional del hábitat. Estas medidas son utilizadas para medir los efectos de panoramas hidrológicos y del uso de tierras frente a los panoramas antes del desarrollo urbano.

\section{Modelos de Decisión}

Los modelos ecológicos proveen datos de salida esenciales para evaluar los cambios de hábitat a nivel de paisaje, pero no los proveen de una manera que: (1) permita evaluaciones colectivas de los cambios de hábitat, o que (2) permita hacer decisiones a partir de múltiples evaluaciones. El análisis de decisiones provee herramientas para formular y evaluar sistemáticamente múltiples criterios y explicar por qué (bajo varias preferencias conflictivas entre sí) una decisión en particular fue tomada (Lahdelma y otros, 2000).

La figura 4 presenta una relación procesal entre evaluaciones científico-técnicas y la creación de políticas o normativas en un cuadro de manejo adaptativo. Los modelos conceptuales son una herramienta inicial efectiva para la identificación grupal de las fuentes de los estresores y de los nexos a los atributos en el ambiente. Los criterios, o medidas de rendimiento, son seleccionados como valores mensurables de atributos identificados, y son utilizados para evaluar el éxito de los planes implementados. Por ejemplo, si un atributo del ambiente natural es la estructura y función de la comunidad de ostras, entonces los criterios para ese atributo pueden ser el crecimiento, enfermedad, mortalidad y reclutamiento de las ostras. Los científicos colocan valores esperados en estos criterios bajo panoramas de cambios ambientales alternos. Esta tarea usualmente se logra mediante el modelaje ecológico. Los encargados de la toma de decisiones luego determinan la importancia de cada uno de estos criterios y utilizan esta información para evaluar diferentes alternativas. Los modelos de decisión ayudan en la comparación y evaluación de alternativas y también pueden ayudar a los encargados de la toma de decisiones a identificar conflictos entre objetivos y a conceptualizar nuevas alternativas que minimicen estos conflictos (Ozernoy, 1984). Una vez que se ha seleccionado e implementado una alternativa, el cambio ambiental esperado es comparado con las condiciones reales mediante monitoreo y experimentación dirigida, lo que puede llevar nuevamente a reevaluaciones de los criterios y de los planes implementados.

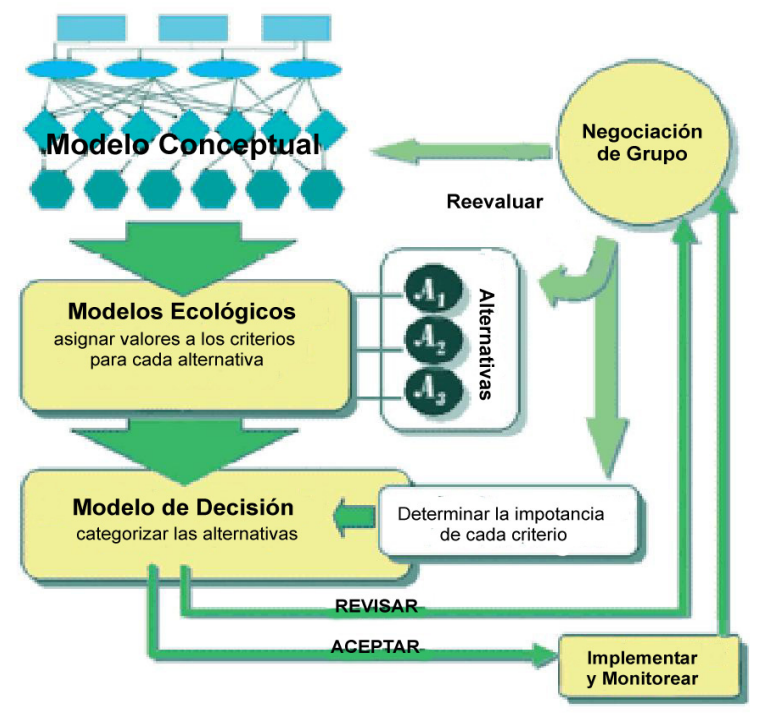

Figura 4. El proceso de soporte de decisión.

Los análisis de decisión de criterios múltiples sirven para que las agencias definan asuntos, propongan soluciones alternas y desarrollen criterios mensurables para evaluar el rendimiento de cada alternativa. Las herramientas de decisión y los métodos gráficos ayudan a proveer una examinación crítica y cuidadosa del proceso. En el sur y el suroeste de Florida, el personal de recursos naturales ha demostrado, mediante una encuesta preliminar acerca del soporte de decisión, la 
necesidad existente de propuestas de evaluación que ayuden a estructurar el proceso de decisión (Pearlstine y otros, 2003).

\section{Referencias Bibliográficas}

Cox, J., Kautz, R., MacLaughlin, M. y Gilbert, T., 1994, Closing the gaps in Floridas wildlife habitat conservation system: Tallahassee, Florida, Office of Environmental Services, Florida Fish and Wildlife Conservation Commission.

Kingston, R., Carver, S., Evans, A. y Turton, I., 2000, Web-based public participation geographical information systems> An aid to local environmental decision-making: Computers, Environment and Urban Systems v. 24, p. 109-125.

Landhelma, R., Salminen, P. y Hokkanen, J., 2000, Using multicriteria methods in environmental planning and management: Environmental Management, v. 26, p. 595-605.

Ozernoy, V., 1984, Gerating alternatives in multiple criteria decision making problems: A survey; en Y. Haimes y V. Chankong (eds.), Decision Making with Multiple Objectives: New York, Springer-Verlag.

Pearlstine, L.G. y Mazzotti, F.J., 2003, A spatially-explicit decision support system for Everglades risk assessment and restoration: An annual report to the U.S. Geological Survey Florida Integrated Science Center: Fort Lauderdale, Florida, Fort Lauderdale Research and Education Center, University of Florida.

Rauscher, H.M., 1999, Ecosystem management decision support for Federal forests in the United States: A review: Forest Ecology and Management, v. 114, p. 173-197.

Tischendorf, L. y Fahrig, L., 2000, On the usage and measurement of landscape connectivity: Oikos, v. 90, p. 7-19.

U.S. Department of the Interior, 2004, Science plan in support of ecosystem restoration, preservation, and protection in South Florida: Accessed on August 26, 2004 at HYPERLINK "http://sofia.usgs.gov/publications/reports/doi- science-plan/" http://sofia.usgs.gov/publications/reports/doi-scienceplan/ 